\begin{tabular}{|c|l|}
\hline Title & ADSORPTION OF GAS MIX TURES ON HETEROGENEOUS SOLID SURFACES \\
\hline Author(s) & JARONIEC, M.; RUDZI SKI, W. \\
\hline Citation & JOURNAL OF THE RESEARCH INSTITUTE FOR CA TALY SIS HOKKA IDO UNIVERSITY, 25(3), 197-210 \\
\hline Issue Date & 1978-03 \\
\hline Doc URL & http://hdl.handle.net/2115/25036 \\
\hline Type & bulletin (article) \\
\hline File Information & 25(3)_P197-210.pdf \\
\hline
\end{tabular}

Instructions for use 


\title{
ADSORPTION OF GAS MIXTURES ON HETEROGENEOUS SOLID SURFACES
}

\author{
By \\ M. JARONIEC ${ }^{*)}$ and W. RUdZIŃSKI*) \\ (Received March 15, 1977)
}

\begin{abstract}
The present state of the studies on adsorption from $n$-component gas mixtures on heterogeneous solid surfaces has been presented in this paper. Using the generalized integral equation, equations of adsorption isotherms and isosteric adsorption heats have been derived. The authors have discussed the simplified integral equation and its application to derivation of analytical equations describing adsorption equilibria.
\end{abstract}

\section{Introduction}

A considerable progress is to be recorded in theoretical description of the adsorption of one component on heterogeneous surfaces. A large number of papers have been published on this subject. However, simultaneous adsorption from a gas mixture of several components remains still open. Although the first studies on adsorption from gas mixtures were started 100 years ago, ${ }^{1)}$ the literature concerning this subject is scanty. The reason of this situation is great difficulties cropping up both in experimental and theoretical study of adsorption from gas mixtures.

In the present studies on adsorption of gas mixtures, the behaviour of binary gas mixtures on homogeneous solid surfaces has been discussed. The earlier papers concerning this problem were discussed in the monographies of Young and Crowell, ${ }^{2}$ and Ponec, Knor and Cerny. ${ }^{3)}$ A rich source of recent papers concerning adsorption of gas mixtures, particularly the Soviet ones, are the materials of the 3rd Conference organized in the Soviet Union, which have been published as a book. ${ }^{4)}$ Moreover, adsorption of gas mixtures on homogeneous surfaces was the subject of a number of reviews. ${ }^{\text {b 8) }}$

Much fewer papers were published on adsorption of gas mixtures on

*) Department of Theoretical Chemistry, Institute of Chemistry, M. Curie-Skłodowska University, 20031 Lublin, Nowotki 12, Poland. 
heterogeneous solid surfaces. No synthetic work on this problem has appeared yet. The purpose of this paper is a critical review of the available papers concerning adsorption from gas mixtures on heterogeneous solid surfaces, which is based mainly on our studies.

\section{General Considerations}

The theory of physical adsorption from $n$-component gas mixtures can be formulated in a clear and simple by means of vector notation. Thus, all functions characterizing the adsorption of $n$-component mixtures will be provided with the index $\boldsymbol{n}$, where $\boldsymbol{n}=(1,2, \cdots, n)$ is an $n$-dimensional vector. Moreover, the pressure vector $\boldsymbol{p}=\left(p_{1}, p_{2}, \cdots, p_{n}\right)$ and that of adsorption energy $\boldsymbol{\varepsilon}=\left(\varepsilon_{1}, \varepsilon_{2}, \cdots \cdots, \varepsilon_{n}\right)$ will also be introduced. Component $p_{i}$ of the vector $\boldsymbol{p}$ denotes the partial pressure of the $i$-th gas in the mixture, whereas component $\varepsilon_{i}$ of the vector $\varepsilon$ denotes the adsorption energy of the $i$-th gas. Then $\Theta_{\boldsymbol{n}}(\boldsymbol{p}, \boldsymbol{\varepsilon})$ and $\vartheta_{\boldsymbol{n}}(\boldsymbol{p})$ denote the total adsorption isotherms for $n$-component gas mixtures on homogeneous and heterogeneous surfaces, respectively; $\chi_{n}(\varepsilon)$ is the $n$-dimensional energy distribution characterizing global heterogeneity of the adsorbent surface; whereas $\Delta_{n}$ denotes the $n$-dimensional region in which the adsorption energies of particular components are contained. In the notation presented here $\Theta_{i}\left(p_{i}, \varepsilon_{i}\right), \vartheta_{i}\left(p_{i}\right), \chi_{i}\left(\varepsilon_{i}\right)$ and $\Delta_{i}$ concern the adsorption of a single component " $i$ ". In doubtless casses index " $i$ " will be omitted.

One more thing to be mentioned is notation for partial adsorption isotherms. The symbols : $\Theta_{i(\boldsymbol{n})}(\boldsymbol{p}, \boldsymbol{\varepsilon})$ and $\vartheta_{i(\boldsymbol{n})}(\boldsymbol{p})$ are proposed for unambigous notation of partial adsorption isotherms; these symbols denote partial isotherms of the $i$-th gas from $n$-component mixture. The sum of partial isotherms gives the total adsorption isotherm:

$$
\vartheta_{\boldsymbol{n}}(\boldsymbol{p})=\sum_{i=1}^{n} \vartheta_{i(\boldsymbol{n})}(\boldsymbol{p})
$$

or

$$
\Theta_{n}(\boldsymbol{p}, \varepsilon)=\sum_{i=1}^{n} \Theta_{i(n)}(\boldsymbol{p}, \varepsilon)
$$

In the case of multilayer adsorption with the symbols $\Theta$ and $\vartheta$ superscript "M" will be added.

The basic equations for adsorption isotherms of $n$-component gas mixtures on homogeneous surfaces will be discussed in short. They will be needed in mathematical formulation of adsorption from gas mixtures on 
Adsorption of Gas Mixtures on Heterogeneous Solid Surfaces

heterogeneous surfaces. The most popular equation of this kind is the generalized one of LANGMUIR :9)

$$
\Theta_{n}(\boldsymbol{p}, \varepsilon)=\frac{\boldsymbol{a} \cdot \boldsymbol{p}}{1+\boldsymbol{a} \cdot \boldsymbol{p}}
$$

where $\boldsymbol{\alpha}=\left(a_{1}, a_{2}, \cdots, a_{n}\right)$, whereas $\boldsymbol{a} \cdot \boldsymbol{p}=\sum_{i=1}^{n} a_{i} p_{i}$ is scalar product of the vectors $\boldsymbol{a}$ and $\boldsymbol{p}$. The components of the vector $\boldsymbol{a}$ are LANGMUIR constants characterizing the adsorption of single gases. They are defined by means of the following equation :

$$
a_{i}=\frac{1}{K_{i}(T)} \cdot \exp \left(\frac{\varepsilon_{i}}{R T}\right)
$$

where $K_{i}$ is the constant connected with molecular partition functions for molecules of the $i$-th component from the first adlayer.

The extension of the BET model to adsorption of gas mixtures was dealt by many authors: Hili, ${ }^{10,11}$ ARnold, ${ }^{12)}$ Bussey, ${ }^{13)}$ and Gonzalez and Holland. ${ }^{14,15)}$ Hill's equation, ${ }^{10)}$ statistically derived by Bussey, ${ }^{13)}$ is very complicated and practically useless from the point of view of the adsorption on heterogeneous surfaces. Moreover, its poor agreement with the experimental data for homogeneous surfaces ${ }^{12)}$ does not predict favourable results for heterogeneous surfaces.

An interesting equation for bilayer adsorption of gas mixtures on homogeneous surfaces was proposed by Gonzalez and Holland :14,15)

$$
\Theta_{\boldsymbol{n}}^{\mathrm{M}}(\boldsymbol{p}, \boldsymbol{\varepsilon})=(1+\boldsymbol{b} \cdot \boldsymbol{p}) \frac{\boldsymbol{a} \cdot \boldsymbol{p}}{1+\boldsymbol{a} \cdot \boldsymbol{p}}
$$

Above, $\boldsymbol{b}=\left(b_{1}, b_{2}, \cdots, b_{n}\right)$ characterizes the formation of a mixed second adlayer. The components $b_{i}$ of the vector $\boldsymbol{b}$ refer to adsorption of single gases. Recently, the model of GoNzalez and Holland was discussed again ${ }^{16)}$ with particular consideration of the simplifying assumptions. It appears that Eq. (5) should work well only in the case of adsorption of mixtures with similar components.

Lately generalized equation of Jovanović was used for interpretation of experimental adsorption isotherms of gas mixtures $:^{17 \sim 19)}$

$$
\Theta_{\boldsymbol{n}}(\boldsymbol{p}, \boldsymbol{\varepsilon})=1-\exp (-\boldsymbol{a} \cdot \boldsymbol{p}) .
$$

Its analogue for bilayer adsorption has the following form :20)

$$
\Theta_{\boldsymbol{m}}^{\mathrm{M}}(\boldsymbol{p}, \varepsilon)=\exp (\boldsymbol{b} \cdot \boldsymbol{p})[1-\exp (-\boldsymbol{a} \cdot \boldsymbol{p})] \text {. }
$$

It is not difficult to check that for low partial pressures Eqs. (6) and (7) are reduced to Eqs. (3) and (5), respectively. 


\section{JARONIEC and W. RUDZIŃSKI}

To conclude this section we shall mention other approaches to the description of adsorption from gas mixtures. The most popular approach is the method developed by Myers and Prausnitz. ${ }^{21}$ In this method the adsorption phase is treated as ideal ${ }^{21 \sim 23)}$ or non-ideal ${ }^{24 \sim 27)}$ surface solution. Such a point of view was also widely applied by Bering and Serpinsky. ${ }^{28,29)}$ The above method has already been often used for interpretation of experimental data. ${ }^{24 \sim 27,30 \sim 32)}$

In monography of Young and CROWELL ${ }^{2)}$ the first papers on mobile adsorption of gas mixtures have been reported. The paper of Ivanov and MARTINOV $^{33)}$ is continuation of this problem. Also, the extension of the model of the associated adsorbate ${ }^{34)}$ to adsorption from gas mixtures ${ }^{35 \sim 37}$ promises to be extremely interesting. This model leads to interesting equations describing monolayer adsorption with interactions as well as multilayer adsorption on homogeneous surfaces.

\section{Generalized Integral Equation in Adsorption of Gas Mixtures}

Let us consider the adsorption of $n$-component gas mixtures on heterogeneous surfaces. The surface of the adsorbent is assumed to be composed of many different homogeneous patches. For adsorption of $n$-component mixture, every patch of the surface is characterized by adsorption energies: $\varepsilon_{1}, \varepsilon_{2}, \cdots, \varepsilon_{n}$, or shorter by vector $\varepsilon$. However, heterogeneity of the adsorbent surface is quantitatively characterized by $n$-dimensional function $\chi_{n}(\varepsilon)$, normalized to unity :

$$
\int_{\Delta_{n}} \chi_{n}(\varepsilon) \mathrm{d} \varepsilon=1
$$

Then, the expression $\chi_{n}(\varepsilon) \mathrm{d} \varepsilon$ (were $\mathrm{d} \varepsilon=\mathrm{d} \varepsilon_{1} \mathrm{~d} \varepsilon_{2} \cdots \mathrm{d} \varepsilon_{n}$ ) denotes a fraction of all adsorption sites on the adsorbent surface, which have their adsorption energies from the region $(\varepsilon, \varepsilon+\mathrm{d} \varepsilon)$. Because, heterogeneity of the adsorbent surface in adsorption of gas mixtures is characterized by $n$-dimensional function $\chi_{n}(\varepsilon)$, the total adsorption isotherm is expressed by means of a multiple integral : $: 38,39$ )

$$
\vartheta_{n}(\boldsymbol{p})=\int_{\Delta_{n}} \Theta_{n}(\boldsymbol{p}, \varepsilon) \chi_{n}(\varepsilon) \mathrm{d} \varepsilon,
$$

where $\Theta_{n}(\boldsymbol{p}, \varepsilon)$ is the so-called local isotherm describing the adsorption on a homogeneous patch of the surface with the energy vector $\varepsilon$. In Eq. (9) for local adsorption isotherm $\Theta_{\boldsymbol{n}}(\boldsymbol{p}, \boldsymbol{\varepsilon})$, Eq. $(3)^{38,39)}$ or $\mathrm{Eq} .(6)^{18,40)}$ can be used.

The generalization of Eq. (9) for multilayer adsorption from gas mixtures 
was discussed in the papers. ${ }^{41,42)}$ The equations (5) and (7) for bilayer adsorption on homogeneous surfaces discussed in section I possess a product form :

$$
\Theta_{\boldsymbol{n}}^{\mathrm{M}}(\boldsymbol{p}, \boldsymbol{\varepsilon})=g_{\boldsymbol{n}}(\boldsymbol{p}) \cdot \Theta_{\boldsymbol{n}}(\boldsymbol{p}, \boldsymbol{\varepsilon}) .
$$

Above, $\Theta_{n}$ is the monolayer adsorption isotherm, whereas function $g_{\boldsymbol{n}}(\boldsymbol{p})$ describes the formation of a multilayer. Introducing Eq. (10) into the following integral equation, describing multilayer adsorption on heterogeneous surfaces :

$$
\vartheta_{n}^{\mathrm{M}}(\boldsymbol{p})=\int_{\Delta_{m}} \Theta_{n}^{\mathrm{M}}(\boldsymbol{p}, \varepsilon) \chi_{n}(\varepsilon) \mathrm{d} \varepsilon,
$$

we obtain

$$
\vartheta_{n}^{\mathrm{M}}(\boldsymbol{p})=g_{n}(\boldsymbol{p}) \cdot \int_{\Delta_{n}} \Theta_{n}(\boldsymbol{p}, \varepsilon) \chi_{n}(\varepsilon) \mathrm{d} \varepsilon=g_{\boldsymbol{n}}(\boldsymbol{p}) \cdot \vartheta_{n}(\boldsymbol{p}) .
$$

As a result the following forms of bilayer adsorption isotherms are obtained:

$$
\begin{aligned}
& \vartheta_{\boldsymbol{n}}^{M}(\boldsymbol{p})=(1+\boldsymbol{b} \cdot \boldsymbol{p}) \cdot \vartheta_{\boldsymbol{n}}^{\mathrm{L}}(\boldsymbol{p}) \\
& \vartheta_{\boldsymbol{n}}^{M}(\boldsymbol{p})=\exp (\boldsymbol{b} \cdot \boldsymbol{p}) \cdot \vartheta_{\boldsymbol{n}}^{\mathrm{J}}(\boldsymbol{p}) .
\end{aligned}
$$

The former is generated by Eq. (5), whereas the latter by Eq. (7). Above, $\vartheta_{\boldsymbol{n}}^{\mathrm{L}}(\boldsymbol{p})$ and $\vartheta_{\boldsymbol{n}}^{\mathrm{J}}(\boldsymbol{p})$ denote monolayer adsorption isotherms obtained from Eq. (9), when the local isotherm is represented by Eq. (3) or Eq. (6), respectively.

\section{Numerical and Analytical Solutions of Generalized Integral Equation}

Adsorption of an $n$-component gas mixture can be calculated numerically from Eq. (9) if the functions $\Theta_{\boldsymbol{n}}(\boldsymbol{p}, \boldsymbol{s})$ and $\chi_{\boldsymbol{n}}(\boldsymbol{\varepsilon})$ are known. For local isotherm Eq. (3) or Eq. (6) is usually assumed, whereas for the function $\chi_{n}(\varepsilon)$ gaussian $n$-dimensional distribution is assumed : $:^{99}$

$$
\chi_{\boldsymbol{n}}(\varepsilon)=\frac{1}{(2 \pi)^{\frac{n}{2}}(\operatorname{det} \boldsymbol{D})^{\frac{1}{2}}} \exp \left[\frac{\sum_{i, j=1}^{n} D_{i j}\left(\varepsilon_{i}-\bar{\varepsilon}_{i}\right)\left(\varepsilon_{j}-\bar{\varepsilon}_{j}\right)}{-2 \operatorname{det} \boldsymbol{D}}\right],
$$

or $n$-dimensional log-normal distribution:

$$
\chi_{\boldsymbol{n}}(\varepsilon)=\frac{1}{(2 \pi)^{\frac{n}{2}}\left(\operatorname{det} \boldsymbol{D}^{*}\right)^{\frac{1}{2}}} \exp \left[\frac{\sum_{i, j=1}^{n} D_{i j}^{*} \ln \left(\varepsilon_{i} / \bar{\varepsilon}_{i}\right) \ln \left(\varepsilon_{j} / \bar{\varepsilon}_{j}\right)}{-2 \operatorname{det} \boldsymbol{D}^{*}}\right],
$$

where $\boldsymbol{D}=\left[d_{i j}\right]_{i, j=1,2, \cdots, n}$ and $\boldsymbol{D}^{*}=\left[d_{i j}^{*}\right]_{i, j=1,2, \ldots, n}$ are two-dimensional matrices ; 
$D_{i j}$ and $D_{i j}^{*}$ are algebraical components of the matrix $\boldsymbol{D}$ and $\boldsymbol{D}^{*}$ for the elements $d_{i j}$ and $d_{i j}^{*}$, respectively; $\bar{\varepsilon}=\left(\bar{\varepsilon}_{1}, \bar{\varepsilon}_{2}, \cdots, \bar{\varepsilon}_{n}\right)$ is the vector characterizing the average surface patch. The parameters $d_{i i}$ and $d_{i i}^{*}(i=1,2, \cdots, n)$ are a measure of the heterogeneity of the surface with regard to molecules of the $i$-th gas. These parameters characterize the adsorption of single gases and they can be evaluated from pure-gas adsorption isotherms. However, the parameters $d_{i j}$ and $d_{i j}^{*}(i \neq j ; i, j=1,2, \cdots, n)$ in the matrices $\boldsymbol{D}$ and $\boldsymbol{D}^{*}$ are dependent upon the kind of the gas mixture and adsorbent surface.

In the paper ${ }^{39)}$ two-dimensional gaussian distribution was applied to interpretation of the experimental adsorption isotherm of ethane-ethylene mixture on Nuxit-AL.43) Hoory and PrausnItz, ${ }^{24)}$ generalizing the method of MYERs and Prausnitz ${ }^{21}$ for adsorption of binary mixtures on heterogeneous surfaces, also used the gaussian two-dimensional distribution.

Now, the analytical solution of Eq. (9) will be discussed. It appears that analytical solutions are not possible for the local isotherms (3) and (6) as well as for more real energy distribution functions. ${ }^{40}$ Only in the case of Jovanović Eq. (6) and a special form of the function $\chi_{n}(\varepsilon)$ the analytical solutions of Eq. (9) are possible. Assuming for function $\chi_{n}(\varepsilon)$ the following form :

$$
\chi_{n}(\varepsilon)=\prod_{i=1}^{n} \chi_{i}\left(\varepsilon_{i}\right)
$$

and using Jovanović Eq. (6) for the local isotherm, we obtain from Eq. (9):

$$
\begin{aligned}
\vartheta_{\boldsymbol{u}}(\boldsymbol{p}) & =1-\prod_{i=1}^{n}\left[\int_{\Delta_{i}} \exp \left(-a_{i} p_{i}\right) \cdot \chi_{i}\left(\varepsilon_{i}\right) d \varepsilon_{i}\right] \\
& =1-\prod_{i=1}^{n}\left[1-\vartheta_{i}\left(p_{i}\right)\right],
\end{aligned}
$$

were $\vartheta_{i}\left(p_{i}\right)$ is an arbitrary adsorption isotherm of the $i$-th gas, generated by the Jovanović equation. The analytical expressions for $\vartheta_{i}\left(p_{i}\right)$ can be taken from MisRA's paper, ${ }^{44)}$ who derived the isotherm equations for adsorption of single gases by assuming exponential and constant energy distribution for $\chi_{i}\left(\varepsilon_{i}\right)$ and the model of Jovanovic for local adsorption. The most interesting equation is obtained for exponential distribution; it is the equation of FREUNDLICH isotherm. Applying this equation to Eq. (18) we obtain

$$
\vartheta_{\boldsymbol{n}}(\boldsymbol{p})=1-\prod_{i=1}^{n}\left[1-K_{i}^{*}\left(p_{i}\right)^{c_{i}}\right]
$$

where $K_{i}^{*}$ and $c_{i}$ are FREUNDLICH constants of the $i$-th gas, and they can be evaluated from pure-gas adsorption data. In the paper, ${ }^{40)} \mathrm{Eq}$. (19) was tested on several adsorption systems: argon-oxygen on cesium iodide, ${ }^{45)} \mathrm{ni}$ - 
Adsorption of Gas Mixtures on Heterogeneous Solid Surfaces

trogen-carbon oxide on cesium iodide ${ }^{45)}$ and xenon-carbon dioxide on aluminium oxide. ${ }^{46)}$

It is of interest that the equation of adsorption isotherm for two-component mixtures obtained by RogINSKY, ${ }^{47}$ apart from the use of another approximation, is a peculiar case of Eq. (18).

\section{Thermodynamic Relations for Gas Mixtures on Heterogeneous Surfaces}

The partition function $Q_{\boldsymbol{n}\left(N_{k}\right)}$ for $n$-component monolayer on heterogeneous surfaces, assuming distribution $N_{i_{k}}(i=1,2, \cdots, n)$ among $\omega_{k}$ sites, may be represented by the following expression $:^{48}$

$$
Q_{n\left(N_{k}\right)}=\prod_{k} \frac{\omega_{k} ! \prod_{i}\left(q_{i_{k i}}^{\mathrm{s}}\right)^{N_{i_{k}}}}{\left[\prod_{i}\left(N_{i_{k}} !\right)\right]\left[\omega_{k}-\sum_{i} N_{i_{k}}\right] !}, \quad i=1,2, \cdots, n
$$

where $\boldsymbol{n}$ and $\boldsymbol{k}$ are $n$-dimensional vectors : $\boldsymbol{n}=(1,2, \cdots, n)$ and $\boldsymbol{k}=\left(\boldsymbol{k}_{1}, \boldsymbol{k}_{2}, \cdots\right.$, $k_{n}$ ). The components of $\boldsymbol{n}$ characterize the gas mixture, whereas the components of $k$ define an adsorption patch. In Eq. (20) $q_{i_{k_{i}}}^{\mathrm{s}}$ is defined by the following relation:

$$
q_{i_{i i}}^{\mathrm{s}}=J_{i_{k_{i}}}(T) \exp \left(\frac{\varepsilon_{i_{k_{i}}}}{k_{\mathrm{B}} T}\right) \approx J_{i}(T) \exp \left(\frac{\varepsilon_{i_{k_{i}}}}{k_{\mathrm{B}} T}\right), i=1,2, \cdots, n
$$

and other symbols have the usual meaning: $k_{\mathrm{B}}$ Boltzmann constant; $\omega_{k}$ number of the sites having the adsorption energies $\varepsilon_{1_{k i}}, \varepsilon_{2_{k_{2}}}, \cdots, \varepsilon_{n_{k_{n}}} ; \omega=\sum_{k} \omega_{k}$ number of all adsorption sites; $N_{i_{k}}$ number of molecules of the $i$-th ${ }^{k}$ gas adsorbed on the $k$-th surface patch : $N_{m}=\sum_{i=1}^{n} \sum_{k} N_{i_{k}}$ number of the molecules adsorbed on the whole surface; $J_{i_{i}}(T)$ partition function of adsorbed isolated molecule of the $i$-th gas on adsorption sites having the energy $\varepsilon_{i_{k_{i}}}$ Eq. (20) is valid if $\omega_{k}$ and $N_{i_{k}}$ for all values of $k$ and $i$ are large numbers, and also $\sum_{i=1}^{n} N_{i_{k}} \leqslant \omega_{k}$. The total partition function $Q_{n}$ is the sum of the partition functions $Q_{m\left(N_{k}\right)}$ :

$$
Q_{n}=\sum_{k} Q_{n}\left(N_{k}\right)
$$

Further, application of the maximum term method ${ }^{49)}$ leads to generalized integral equation (9) with local adsorption isotherm of LANGMUIR type.

Using the maximum term of expression (22) for the partition function $Q_{n}$, the various thermodynamic functions for localized mixed monolayer on heterogeneous surfaces can be obtained :48) 
M. JARONIEC and W. RUDZIŃSKI

$$
\begin{aligned}
& A_{n}=\omega \int_{\Delta_{n}}\left[\mu^{\mathrm{G}} \Theta_{n}+k_{\mathrm{B}} T \ln \left(1-\Theta_{n}\right)\right] \chi_{n}(\varepsilon) \mathrm{d} \varepsilon \\
& E_{n}=\omega \int_{\Delta_{n}}\left(E_{n} \Theta_{n}\right) \chi_{n}(\varepsilon) \mathrm{d} \varepsilon \\
& S_{n}^{\mathrm{c}}=-k_{\mathrm{B}} \omega \int_{\Delta_{n}}\left[\Theta_{n} \ln \Theta_{n}+\left(1-\Theta_{n}\right) \ln \left(1-\Theta_{n}\right)\right] \chi_{n}(\varepsilon) \mathrm{d} \varepsilon \\
& S_{n}^{\mathrm{nc}}=k_{\mathrm{B}} \omega \int_{\Delta_{n}}\left[S_{n}^{\mathrm{nc}} \Theta_{n}\right] \chi_{n}(\varepsilon) \mathrm{d} \varepsilon \\
& Q_{i}^{\mathrm{st}}=\frac{\int_{\Delta_{n}} q_{i}^{\mathrm{st}} \chi_{n}(\varepsilon)\left[\frac{\partial \Theta_{n}(p, \varepsilon)}{\partial \ln p_{i}}\right]_{T, p_{j \neq i}} \mathrm{~d} \varepsilon}{\int_{\Delta_{n}} \chi_{n}(\varepsilon)\left[\frac{\partial \Theta_{n}(\boldsymbol{p}, \varepsilon)}{\partial \ln p_{i}}\right]_{T, p_{j \neq i}}^{\mathrm{d} \varepsilon}}
\end{aligned}
$$

where $A_{n}=-k_{\mathrm{B}} T \ln Q_{n}$ Helmholtz free energy; $E_{n}=k_{\mathrm{B}} T^{2}\left[\frac{\partial \ln Q_{n}}{\partial T}\right]$ energy of the system; $S_{n}^{\mathrm{c}}$ configurational entropy; $S_{n}^{\mathrm{nc}}$ nonconfigurational entropy; $S_{n}=S_{n}^{\mathrm{c}}+S_{n}^{\mathrm{nc}}=\left(E_{n}-A_{n}\right) / T$ entropy of the system; $Q_{i}^{\text {st }}$ and $q_{i}^{\text {st }}$ are total and local isosteric heats of the $i$-th component, respectively. The other symbols from Eqs. $(23) \sim(27)$ are defined as follows :

$$
\begin{aligned}
\mu^{G} & =\left(\mu_{1}^{G}, \mu_{2}^{G}, \cdots, \mu_{n}^{G}\right) \\
\Theta_{n} & =\left(\Theta_{1(n)}, \Theta_{2(n)}, \cdots, \Theta_{n(n)}\right) \\
\ln \Theta_{n} & =\left(\ln \Theta_{1(n)}, \ln \Theta_{2(n)}, \cdots, \ln \Theta_{n(n)}\right) \\
\boldsymbol{E}_{n} & =\left(E_{1}, E_{2}, \cdots, E_{n}\right) \\
\boldsymbol{S}_{n}^{n \mathrm{c}} & =\left(S_{1}^{\mathrm{nc}}, S_{2}^{\mathrm{nc}}, \cdots, S_{n}^{\mathrm{nc}}\right)
\end{aligned}
$$

where

$$
\begin{aligned}
\Theta_{i(\boldsymbol{n})} & =q_{i}^{*} /\left(1+\sum_{j=1}^{n} q_{j}^{*}\right) \\
q_{i}^{*} & =q_{i}^{\mathrm{s}} \exp \left(\frac{\mu_{i}^{\mathrm{G}}}{k_{\mathrm{B}} T}\right)=q_{i}^{\mathrm{s}} \exp \left(\frac{\mu_{i}^{0 \mathrm{G}}}{k_{\mathrm{B}} T}\right) p_{i} ; \quad i=1,2, \cdots, n
\end{aligned}
$$

and

$$
\begin{aligned}
S_{i}^{\mathrm{nc}} & =T \cdot \frac{\partial \ln J_{i}}{\partial T}+\ln J_{i} \\
E_{i} & =k_{\mathrm{B}} T^{2} \frac{\partial \ln J_{i}}{\partial T}-\varepsilon_{i} .
\end{aligned}
$$

In the above, $\mu_{i}^{G}$ is the chemical potential of the $i$-th component in the gas phase; $S_{i}^{\text {nc }}$ and $E_{i}$ are nonconfigurational part of the entropy and the energy for the adsorption of molecule " $i$ " on a patch with the energy of adsorp- 
tion of that molecule of $\varepsilon_{i}$ respectively. In the paper ${ }^{50)}$ the isosteric adsorption heats for the components of binary gas mixtures were calculated according to Eq. (27) by using the experimental adsorption parameters. The calculations carried out there concerned the adsorption of xenon from xenoncarbon dioxide mixture on aluminium oxide ${ }^{46)}$ and that of propylene from propylene-propane mixture on Nuxit-AL. ${ }^{43)}$ For local adsorption, the Jovanović Eq. (6) was used, whereas for the energy distribution function-exponential distribution in the case of xenon-carbon dioxide on aluminium oxide and gaussian distribution in the case of propylene-propane on Nuxit-AL were used.

\section{Simplified Integral Equation in Adsorption of Gas Mixtures}

In description of the adsorption from gas mixtures on heterogeneous surfaces the simplified form of the integral equation (9) was extremely often used. In this section the derivation of the simplified intergral equation for adsorption of gas mixtures will be briefly presented and its more important applications will be discussed.

Let us choose any component of the mixture, which will be compared with other components; let it be component 1 . Assume that relationship between adsorption energy of any component " $i$ " $(i>1)$ and adsorption energy $\varepsilon_{1}$ is known:

$$
\varepsilon_{i}=f_{i}\left(\varepsilon_{1}\right) \quad \text { for } i=2,3, \cdots, n
$$

Then the generalized intergral equation (9) can be transformed to the following from $:^{51}$

$$
\vartheta_{n}(\boldsymbol{p})=\int_{\Lambda_{1}} \Theta_{n}^{*}\left(\boldsymbol{p}, \varepsilon_{1}\right) \chi_{1}\left(\varepsilon_{1}\right) \mathrm{d} \varepsilon_{1}
$$

where

$$
\Theta_{n}^{*}\left(\boldsymbol{p}, \varepsilon_{1}\right)=\Theta_{\boldsymbol{n}}\left(\boldsymbol{p}, \varepsilon_{1}, f_{2}\left(\varepsilon_{1}\right), f_{3}\left(\varepsilon_{1}\right), \cdots, f_{n}\left(\varepsilon_{1}\right)\right)
$$

and

$$
\chi_{1}\left(\varepsilon_{1}\right)=\int_{\Delta_{n}^{*}} \chi_{n}(\varepsilon) \mathrm{d} \varepsilon_{2} \mathrm{~d} \varepsilon_{3}, \cdots, \mathrm{d} \varepsilon_{n} .
$$

In the above, $\Delta_{n}^{*}=\Delta_{2} \times \Delta_{3} \cdots \times \Delta_{n}$ is the $(n-1)$ dimensional integration region. Equation (34), however, defines the so-called "border" energy distribution. Equation (32) has been used by many authors for description of the 


\section{JARONIEC and W. RUDZIŃSKI}

adsorption of binary gas mixtures on heterogeneous surfaces. The first and most advanced theoretical results are due to Roginsky and ToDEs ${ }^{52,53)}$ and GLUECKAUF ${ }^{54)}$. ROGINSKY and TODEs extended the approximate control band method ${ }^{55)}$ to adsorption of binary gas mixtures. They used an integral equation entirely similar to Eq. (32) and assumed the linear relation between adsorption energies :

$$
\varepsilon_{2}=\alpha \varepsilon_{1}+\beta .
$$

Roginsky and Todes have considered the following possibilities: that adsorption energies vary similarly ${ }^{52)}(\alpha>0)$, and that they vary contrariwise ${ }^{53)}$ $(\alpha<0)$. The first case was also considered by Bering and Serpinsky. ${ }^{56}$ GLUECKAUF ${ }^{54}$, to describe the adsorption of binary gas mixtures on heterogeneous surfaces, also used the integral equation (32). The relationship between adsorption energies of both components, assumed by him, is a special case of relation (35), when $\beta=0$. The most interesting result in GlueckauF treatment is FREUNDLICH isotherm for binary gas mixtures, which was earlier obtained by Tompkins and Young. ${ }^{45)}$ This isotherm can be derived on assuming an exponential distribution of adsorption energy, and both gases have the same FREUNDLICH constants $c(0<c<1)$.

Recently, JARONIEC and TóTH ${ }^{\text {67 60) }}$ and also TIEMKIN ${ }^{61)}$ extended the best known adsorption isotherms, i.e., Tó $\mathrm{TH}^{62)}$ and FREUNDLICH ${ }^{63)}$ isotherms, to monolayer adsorption of binary gas mixtures on heterogeneous surfaces. JARONIEC and TбTH considered two cases:

-the adsorbing components behave very similarly on the surface, i.e., the heterogeneity parameters are approximately equal: $m_{1}=m_{2}$ (Тб́тн раrameters) and $c_{1}=c_{2}$ (FREUNDLICH parameters). This condition means that the relationship between adsorption energies of both components is given by Eq. (35) for $\alpha=1.57,58)$

- the adsorbed components show a different behaviour on the surface of the adsorbent ${ }^{59)}$ component 1 is used either for the equation of Tбтн or FREUNDLICH, whereas component 2 shows LANGMUIR behaviour.

In the paper, ${ }^{60)}$ the above cases for bilayer adsorption were discussed. Thermodynamic aspects of this method, on assuming the relationship (35) with $\alpha=1$, were discussed by SNAGovsky. ${ }^{64)}$

As it appears from the literature cited here, the relationship (35) with $\alpha=1$ is often used in theoretical considerations and finds experimental support. ${ }^{57,61,65)}$ For $n$-component mixtures it can be written:

$$
\varepsilon_{i}-\varepsilon_{1}=\beta_{i} \quad \text { for } i=2,3, \cdots, n .
$$

Then, the term $\boldsymbol{a} \cdot \boldsymbol{p}$ occurring in Eqs. (3) and (6) can be presented in a little 
Adsorption of Gas Mixtures on Heterogeneous Solid Surfaces

different form :

$$
\boldsymbol{a p}=a_{1} \boldsymbol{r}_{1} \boldsymbol{p},
$$

where $r_{1}=\left(r_{11}, r_{12}, \cdots, r_{1 n}\right)$ and $r_{11}=1$.

The components of vector $r_{1}$ are defined in the following way:

$$
r_{1 i}=a_{i} / a_{1}=\frac{K_{1}}{K_{i}} \exp \left(\frac{\beta_{i}}{R T}\right) \quad \text { for } i=2,3, \cdots, n
$$

and are invariable for all homogeneous surface patches. Then the integral (32) assumes the following forms :

$$
\vartheta_{n}(\boldsymbol{p})=\int_{\Delta_{1}} \frac{\chi_{1}\left(\varepsilon_{1}\right) \mathrm{d} \varepsilon_{1}}{1+\frac{K_{1}}{\boldsymbol{r}_{1} \boldsymbol{p}} \exp \left(\frac{-\varepsilon_{1}}{R T}\right)}
$$

for Langmuir adsorption isotherm (3), and

$$
\vartheta_{n}(\boldsymbol{p})=\int_{\Delta_{1}}\left\{1-\exp \left[-\frac{\boldsymbol{r}_{1} \boldsymbol{p}}{K_{1}} \exp \left(\frac{\varepsilon_{1}}{R T}\right)\right]\right\} \chi_{1}\left(\varepsilon_{1}\right) \mathrm{d} \varepsilon_{1}
$$

for Jovanović adsorption isotherm (6)

The integral (39) can be brought to STIELTJEs transform by way of conventional transformations, ${ }^{63)}$ and integral $(40)$ can be transformed to LAPLACE transform. ${ }^{66)}$ The solutions of STIELTJEs and LAPLACE transforms are known for many distributions such as : exponential, ${ }^{44,63)}$ DUBININ-RAdushKEVICH, ${ }^{67,68)}$ and other quasigaussian distributions. ${ }^{69,70}$ Thus, the monolayer adsorption isotherms of gas mixtures on heterogeneous surfaces can be obtained from equations of adsorption isotherms of single gases, replacing in them the adsorbate pressure by expression $\boldsymbol{r}_{1} \boldsymbol{p}$. In this way the best known adsorption isotherms of single gases such as: FreundLich, Tóth and DubininRADUSHKEVICH have been generalized to adsorption of $n$-component gas mixtures..$^{57,59,61,65)}$ In the papers ${ }^{57,65)}$ the obtained isotherms have been tested experimentally using adsorption data for hydrocarbon mixtures on Nuxit-AL (charcoal), ${ }^{57)}$ argon-oxygen on cesium iodide, ${ }^{65)}$ and argon-nitrogen on chabazite. ${ }^{65}$ It appears from these studies that these equations describe well the experimental data.

In conclusion, worth mentioning is methods for description of experimental data of adsorption from gas mixtures, which are characterized by stability of one of the parameters; it can be:

1) total pressure of the mixture ${ }^{71,722}$

2) partial pressure of the chosen component ${ }^{73)}$

3) mole fractions of the components in the gas phase $e^{65}$

4) mole fractions of the components in the adsorption phase ${ }^{45)}$ 
M. JARONIEC and W. RUNZIŃSKI

5) adsorption of one of the components. ${ }^{74,75)}$

The stability of the first three parameters can experimentally be controlled for any adsorption system, i.e., adsorption data can be measured with a stable parameter.

The stability of the two last parameters is a characteristic feature of only some adsorption systems. More interesting cases are three first, because the stability of the parameters can be maintained by means of a specially constructed apparatus. Mathematical analysis of experimental data measured with a stable parameter is simpler. This problem has been discussed in details in the papers. ${ }^{45,65,71,75)}$

\section{Acknowledgement}

This research was supported by Polish Academy of Sciences, problem No. 03. 10. 4. 01.09.

\section{References}

1) R. A. SMITh, Proc. Roy. Soc., 12, 424 (1863).

2) D. M. Young and A. D. Crowell, Physical Adsorption of Gases, Butterworths, London 1962, pp. 365-406.

3) V. Ponec, Z. KNoR and S. Cerny, Adsorption on Solids, Butterworths, London 1974, pp. 313-319 and 393-402.

4) Physical Adsorption from Multicomponent Phases, ed. M. M. DUBININ and V. V. SERPINSKY, Nauka, Moscow 1972 (in Russian).

5 ) H. C. VAN NEss, Ind. Eng. Chem. Fundam., 8, 464 (1969).

6) A. NodzEński, Wiadomości Chem. Warsaw, 30, 387 (1976).

7) S. SircAR and A. L. Myers, Chem. Eng. Sci., 28, 489 (1973).

8) M. Bülow, A. GrossmanN and W. Schirmer, Z. Chemie, 12, 161 (1972).

9 ) E. C. Markham and A. F. Benton, J. Am. Chem. Soc., 53, 497 (1931).

10) T. L. Hill, J. Chem. Phys., 14, 268 (1946).

11) T. L. HILL, J. Chem. Phys., 14, 46 (1946).

12) J. R. ARnold, J. Am. Chem. Soc., 71, 104 (1949).

13) B. W. BusSEy, Ind. Eng. Chem. Fundam., 5, 103 (1966).

14) A. J. GonZAlez and C. D. Holland, AIChE, 16, 718 (1970).

15 ) A. J. Gonzalez and C. D. Holland, AIChE, 17, 470 (1971).

16) M. JARoniec, M. Borówko and W. Rudziński, AIChE, 23, 605 (1977).

17) M. JARONIEC, Chem. Zvesti, 29, 512 (1975).

18) M. JARoniec and J. Tóth, Acta Chim. Hung., 91, 153 (1976).

19) V. POPA and E. SEgAL, Rev. Roum. Chim., 21, 977 (1976).

20) M. JARONIEC, Chem. Zvesti, 30, 658 (1976).

21) A. L. Myers and J. M. Prausnitz, AIChE, 11, 121 (1965).

22) S. Sircar and A. L. Myers, Chem. Eng. Sci., 28, 489 (1973). 


\section{Adsorption of Gas Mixtures on Heterogeneous Solid Surfaces}

23) A. J. KidnAy and A. L. MYers, AIChE, 12, 981 (1966).

24 ) S. E. Hoory and J. M. Prausnitz, Chem. Eng. Sci., 22, 1025 (1967).

25) S. E. Hoory and J. M. Prausnitz, Chem. Eng. Progress Symposium Series, 63, 3 (1967).

26) R. O. FRIEDERICH and J. C. Mullins, Ind. Eng. Chem. Fundam., 11, 439 (1972).

27) E. D. SLoAN and J. C. Mullins, Ind. Eng. Chem. Fundam., 14, 347 (1975).

28 ) B. P. BERING and V. V. SERPINSKY, Izv. Akad. Nauk USSR, Ser. Khim., 169 (1972).

$29)$ B. P. Bering and V. V. Serpinsky, Izv. Akad. Nauk USSR, Ser. Khim., 171 (1972).

30) L. R. DORFMAN and R. P. DANNER, AIChE Symposium Series, 71, 30 (1975).

31 G. A. Perfetti and J. P. Wightman, J. Coll. Interface Sci., 55, 252 (1976).

32 ) G. A. Perfetti and J. P. Wightman, Carbon, 13, 473 (1975).

33) Ref. 4, pp. 191-201.

34 ) I. G. Berezin and A. V. Kiselev, J. Coll. Interface Sci., 38, 227 (1972).

35) M. JARONIEC, Vuoto, 9, 36 (1976).

36) M. Jaroniec, Colloid \& Polymer Sci., 256, in press (1978).

37) Borówko, M. JARONIEC and W. Rudziśski, Colloid \& Polymer Sci., 256, in press (1978).

38) M. JARoniec and W. Rudziński, Phys, Letters, 53 A, 59 (1975).

39) M. JARONIEC and W. RUdZIŃSkI, Surface Sci., 52, 641 (1975).

40) M. JARONIEC, J. Coll. Interface Sci., 53, 422 (1975).

41) M. Jaroniec, Phys. Letters, 56 A, 53 (1976).

42) M. Jaroniec, J. Tóth and W. RudzińsKi, Acta Chim. Hung., 94, 35 (1977).

43) L. Szepesy and V. Illes, Acta Chim. Hung., 35, 245 (1963).

44 ) D. N. MisRA, J. Coll. Interface Sci., 43, 85 (1973).

$45)$ F. C. Tompkins and D. M. Young, Trans. Faraday Soc. 47, 96 (1951).

46) M. Lasoń and A. Nodzeński, Ann. Soc. Chim. Polonorum, 47, 591 (1973).

47) Ref. 3, pp. 401.

48) M. JARonIEC, J. Chem. Soc. Faraday II, 73, 933 (1977).

49) T. L. Hill, J. Chem. Phys., 17, 762 (1949).

50) M. Jaroniec, J. Coll. Interface Sci., 59, 371 (1977).

51) M. JARoniec, Ph. D. Thesis, M. Curie-Skłodowska Univ., Lublin 1976.

52 ) S. RogINSKY and O. TODES, Acta Physicochim. USSR, 20, 307 (1945).

53) S. Roginsky and O. Todes, Acta Physicochim. USSR, 20, 696 (1945).

54) E. Glueckauf, Trans. Faraday Soc., 49, 1066 (1953).

55) S. Roginsky and M. I. YANovsky, Izv. Akad. Nauk USSR, 59 (1952).

56) B. P. BERING and V. V. SERPINSKy, Izv. Akad. Nauk USSR, 37 (1953).

57) M. Jaroniec and J. Tóth, Colloid Polymer Sci., 254, 643 (1976).

58) M. JARONIEC, Colloid Polymer Sci., 255, 32 (1977).

59) M. JARoniec, Colloid Polymer Sci., 255, 176 (1977).

60) M. JARONIEC and J. Tóth, Colloid Polymer Sci., 256, in press (1978).

61) M. I. Tiemkin, Kinetika \& Kataliz, USSR, 17, 1461 (1975).

62 ) J. Tóth, Acta Chim. Hung., 69, 311 (1971).

63 ) D. N. Misra, J. Chem. Phys., 52, 5499 (1975).

$64)$ S. SNAGOVSKY, Kinetika \& Kataliz, USSR, 17, 1435 (1975). 
65) M. JARoniec, J. NARKIEwicz and W. Rudziński, J. Coll. Interface Sci., in press. (1978).

66) W. RudzIŃSKI and M. JARONIEC, Surface Sci., 42, 552 (1974).

67 ) D. N. MisRa, Surface Sci., 18, 367 (1969).

68) M. JARONIEC, Surface Sci., 50, 553 (1975).

69) R. SIPS, J. Chem. Phys., 18, 1024 (1950).

$70)$ J. Tóth, W. Rudziński, A. WAKSMUNdzki, M. JARONIEC and S. SoKozowski, Acta Chim. Hung., 82, 11 (1974).

71) M. JaRoniec, J. Coll. Interface Sci., 59, 230 (1977).

72) M. JARonIEC, J. Low Temperature Phys., 24, 253 (1976).

73) M. JARONIEC, Vuoto, 9, 57 (1976).

74) M. JARoniec, J. Coll. Interface Sci., 52, 41 (1975).

$75)$ M. JARoniec, Z. phys. Chem., 257, 449 (1976). 